\title{
Gender Roles in Intimate Relationships: Who Initiates and Why?
}

\author{
Natalie Schneider ${ }^{1}$
}

\begin{abstract}
The gender roles of men and women are continuously changing in heterosexual relationships alongside the ever-increasing flexibility and variation of preferences, choice, agency, and individual needs. This paper delves into the role tradition plays between men and women in intimate relationships regarding marriage proposals and surname changes, as well as which sex initiates more when it comes to physical intimacy, emotional intimacy, and long-term commitment.
\end{abstract}

\section{Introduction}

Three primary characteristics of intimate relationships are physical intimacy, including sexual and non-sexual touch; emotional intimacy, including the sharing of thoughts, feelings, and mutual and individual experiences; and commitment, including cohabitation, living apart together relationships, and marriage. Pertinent to these three characteristics of intimate relationships is the act of initiation: who is it, men or women, that initiate more when it comes to a sexual relationship, an emotional connection, and commitment to each other? According to heteronormative stereotypes, it is instinctual to assume that men are the initiators of sex and women the initiators of emotional intimacy. When it comes to commitment, we assume women to be most desirous of commitment while we depend on men to be the proposer of such things like marriage. Sex typically refers to a person's biological traits, distinguishing between male and female, while gender is used to identify how a person relates to him or herself regardless of biological sex traits. For the purpose of this paper, the terms gender and sex are used interchangeably as the information that follows is an analysis of intimate relationships between heterosexual males and females (American Psychological Association, 2020). Also worth noting is the term sociosexuality, also referred to as sociosexual orientation, which is "a dimension of personality that describes people's comfort with

\footnotetext{
${ }^{1}$ MacEwan University, Edmonton, Alberta
} 
and preference for sexual activity in the absence of love or commitment" (American Psychological Association, 2020). We will be exploring the ways in which men and women come together in relationship in three categories: physical intimacy, emotional intimacy, and long-term commitment and who is more likely to initiate each stage. As relationship dynamics continue to change between men and women, where cohabitation, divorce, sex before marriage, women prioritizing careers, and men taking on stronger roles in the household, has the way in which we form our most intimate relationships changed as well? It is my hypothesis that while intimate relationships between men and women have evolved drastically over the last century where rigid gender roles have softened and strict courtship rules have loosened, the essence of male initiation and female receptivity remains the instinctual, preferred method of relating to the opposite sex while coming together to form an intimate heterosexual relationship on the part of both men and women with or without emotional involvement.

\section{Relationship Initiation}

Traditionally, and perhaps stereotypically, men have been the pursuers of women and women the receivers of their attention. In line with this and pertaining to formation of relationship between the sexes, Clark et. al. (1999) describes the initiation of a relationship by either making physical contact with the other or by verbally specifying an interest in beginning a relationship with the other (p. 712). Through a series of interview questions, it was revealed that while women reported having been just as successful as their male counterparts in initiating a romantic relationship, it was men who initiated more frequently (Clark et. al., 1999). Along with higher frequency of male initiation is an overall acceptance, confidence, and motivation to initiate in whatever way they (men) deem appropriate to gain the attention and approval of women (Clark et. al., 1999). On the other hand, women were not only less likely to initiate but were more comfortable taking on a receptive role and were more confident in relying on how they portrayed themselves, primarily physically (Clark et. al., 1999). Receptivity and passivity are often used interchangeably in conversation and literature but are in fact two different ways of being. Much like how male assertiveness and direct communication can be perceived in a negative way, I would like to suggest that receptivity is misrepresented by using it interchangeably with passivity and connotes a negative impression where traditional femininity is, by nature, receptive. Passivity is "a form of adaptation, or maladaptation, in which the individual adopts a pattern of submissiveness, dependence, and retreat into inaction" whereas receptivity is defined as "the period of time when a female is responsive to sexual overtures from a male" (American Psychological Association, 2020).

The study's results showed that direct strategies were employed by men and women, but more so men, and indicated higher ratings of effectiveness and frequency of use in five of the eight factor ratings: Direct communication whether through physical means or otherwise is proficient, potent, open, flirtatious, and uninhibited (Clark et. a., 1999). Passivity on the other hand scored as being the least proficient in initiating intimate relationships (Clark et. al., 1999).

Another strategy of initiation is emotional disclosure: A warm sociability expressing one's personal interests and feelings with others of all ages and genders, seen as a positive trait indicating emotional safety and availability. It is symbolic of the universal and personal mother and is often 
employed more comfortably by women. In a scene where an intimate relationship is just beginning, it signals to the other, often men but not always, a woman's receptivity to him emotionally and often physically. In another study by Clark et. al., the findings were similar in that direct communication patterns and displaying emotional openness were strong catalysts in relationship initiation success indicating that it is not only biological sex but gender-role association that plays into the ways in which men and women communicate a desire for connection (Clark et. al., 1999). These initiation strategies seem to be used for the pursuit of long-term relationships more so than short-term sexual encounters indicating that different strategies other than direct communication and emotional disclosure are utilized to initiate sociosexual relationships such as hookups (Clark et. al., 1999). This is significant: As relationship dynamics and constellations continue to grow and diversify, utilizing these two initiation strategies indicates that the people using them are interested in traditional relational dynamics beginning with direct communication on the part of men, and being reciprocated by emotional disclosure on the part of women.

To initiate means to put something in motion. While direct advances in relationship initiation on the part of men are seen as the first line of communication, there is an argument to be had that women initiate by signaling to men through covert, abstract ways that they themselves are open to being pursued. However, given that openness does nothing to put something into motion and is merely a psychological quality others may pick up on, it is the direct verbal and physical measures that initiate relationships. Although the effectiveness is the same when men and women use direct communication, the increased frequency of men utilizing this technique implies a preference for this gender role to remain as it is.

Anecdotally, I have observed over several years pre-pandemic the initiation of Latin partner dancing in public social settings where leads are most often male and follows are most often women. There is no official rule on who ask who to dance regardless of ethnicity, however, I have observed that it is more common for the males to initiate a dance with a woman. This is done either by using physical gestures only in the form of a head nod towards the dance floor once eye contact has been made, or by offering a hand. Sometimes verbal communication is used only, and most frequently it is a combination of the two: an arm or hand offered, or a gentle touch followed by a question asking for a dance. Sometimes women say no, but more often they say yes. I have witnessed the opposite as well, where a woman will ask a man to dance and repeatedly so with one of my close female friends. This initiation is always verbal, never physical. Sometimes the men say yes, but more often they says no, and while this is an example of social dancing and not a monogamous relationship, it is an initiation nonetheless between a man and a woman indicating a desire for physical intimacy, mutual participation, and emotional expression through the art of dance. Although limited to a dance connected to a culture where men are typically more domineering, it indicates relational dynamic between the sexes where traditional gender roles exist and persist. Worth noting is the frequent disappointment of my female friend upon the rejection of her initiations, and her confusion as to why she is not asked more frequently compared to other women on the scene. By most she would be considered a physically attractive, successful, and independent woman indicating there could be something in the development of her traditionally masculine qualities, such as her assertiveness, that interrupt the natural flow of male initiation and female receptivity. Accordingly, males seem to initiate romantic relationships more often and more easily than females (Eryilmaz et. al., 2011). There are clearly cultural reasons at play for why this 
may be so, and it could also be due to developmental factors, as indicted by Eryilmaz et. al. where the results of their study showed that "males scored significantly higher than females in terms of self-perception, behavioural intimacy, emotional and cognitive intimacy, and romantic verbalization" (2011, p. 597). Their study also showed that it is the attribution of masculine traits found in traditional gender norms that are associated with the initiation of intimate relationships (Eryilmaz et. al., 2011).

\section{Emotional Intimacy}

Emotional intimacy is an important component of intimate relationships, and the relationship initiation is one example: Whether by physical or verbal means, the act of contacting someone of interest expresses a vulnerability on the part of the initiator, a fundamental piece in establishing an emotional connection with another. Continued acts of initiation and reception on the parts of both sexes lead to and often indicate the existence of love. Eventually someone will utter the phrase "I love you" and a failure to do so can indicate relationship dissolution. This phrase is a hallmark sign of emotional intimacy around the world. It is usually spoken once an emotional attachment has been formed, often within the first few months of dating someone, and sometimes much earlier. Emotional expressions such as this hold significant weight in propelling a relationship forward and thus can been seen as an initiatory event specific to emotional intimacy (Harrison et. al., 2011). It signifies commitment, feelings of trust, and an attitude of acceptance. The lack of reciprocation holds the power to derail not only positive romantic interactions gained thus far, but also a failed attempt to move the relationship on to the next stage. As the expected possessor of emotional warmth, it could be anticipated that women are the ones to express this phrase first either genuinely after some time has passed, or less genuinely after just a short while to successfully satisfy one's personal agenda. Although tactics of manipulation scored low in effectiveness according to Clark et. al., (1999) it could be seen as a way for both men and women to secure relationship goals (Harrison et. al., 2011). Where women are conditioned and expected to desire a long-term relationship where commitment is central, men are more readily accepted and expected to pursue multiple years of short-term sexual encounters without any intentions of committing to one person (Harrison et. al., 2011). Either sex could use the words "I love you" as gaining their object of desire. However, the seriousness of these words would likely be effective only on naive females, unaware of manipulative attempts. I would expect equally low effectiveness in using this emotional expression to gain a long-term relationship early on unless there were other tangible, reciprocal rituals that had indicated honesty and potential for reciprocity such as meeting each other's friends and families.

\section{Physical Intimacy}

Pertaining both to long term and short-term relationships, the tendency for men to be more comfortable with sex sooner timewise is reflected in the study done by Harrison et. al. (2011) where the results showed that "women reported a desire to wait longer to have sex" (p. 731). How- 
ever, contrary to popular belief that men separate emotions from sex, this study showed that men expected themselves to be ready to have sex for the first time around the same time in which they knew they were in love, as well as knowing their partner was in love (Harrison et. al., 2011). The research shows that men take first place when it comes to developing feelings for their partners compared to women, and thus are ready for sex before women (Harrison et. al., 2011). This is interesting in that the term feelings can be interpreted in a broad sense and could encompass different types between men and women. Where sociosexuality refers to an absence of feelings, perhaps a better explanation of hookups and other short-term sexual relationships is better described reflecting feelings of attraction, lust, and desire not necessarily companioning deeper feelings of care, love, and emotional attachment. It could also indicate the confusion of love with sexual desire or attraction. Nonetheless, it does indicate a readiness on the part of men for emotional and sexual intimacy before that of women. In fact, "men have been found to take the initiative more than three times that of women during the first year of marriage" and their research revealed that single men were twice as likely to initiate sexual intercourse than women were (Grøntvedt et. al., 2015, p. 516). They also found that men already in monogamous relationships initiated sex three and a half times more often than women (Grøntvedt et. al., 2015). In either case, committed or not, men are more likely to initiate sex. Factors that increased a woman's initiation of sexual intercourse were related to feelings of compatibility, and relationship duration past the two-year mark (Grøntvedt et. al., 2015). Factors that increased male initiation of sexual intercourse were high scores of independence, whereas relational attachment decreased sexual initiation (Grøntvedt et. al., 2015).

\section{Commitment}

Commitment in modern relationships can be defined in a multitude of ways given the variety of relationship constellations. Commitment in polyamorous relationships, for example, can be viewed by two people as remaining dedicated to encouraging each other's personal growth, an inter-dependence in shared domestic life, and as primary support persons throughout life while not limiting sexual activity strictly to each other. In living apart together (LAT) relationships, it is not uncommon to find a high degree of commitment to each other including sexual monogamy, mutual goals for the future, and a high level of desire. These relationships, when centered around a mutual desire to uphold individual responsibilities outside the relationship, can be highly satisfying and supportive to both parties. For the purposes of this paper on traditional in heterosexual relationships and the associated gender norms, I will focus on commitment as seen through the act of marriage.

What I found most interesting while reading on marriage, both the moving towards and dissolving of, were the initiatory practices. It is typically portrayed in popular culture that women seek marriage and wait for the man to propose it. It is not uncommon for females to be involved in the process of engagement to varying degrees in real life (Schweingruber et. al., 2004). Because transitioning into the stage of engagement and marriage is commonly discussed between a couple, the initiatory behaviour of a marriage proposal could be seen as the result of a woman having already initiated a conversation about her personal readiness for marriage. From engagement ring 
preferences, to budgeting for the future, to a strong opinion on asking the father for his blessing, the events leading up to a marriage proposal appear to be a mutual task.

However, according to Schweingruber et. al. (2004), there are three groups of people that display a strong preference for a traditional marriage proposal defined as the male bending on one knee, offering of a diamond ring, and his request for her hand in marriage. From this standpoint, engagement seems to be initiated strictly by males. The three groups that prefer this method are women, religious persons, and younger people, the latter two of which include both sexes (Schweingruber et. al., 2004). The study by Robnett et. al. (2013) confirms that both sexes prefer traditional marriage proposals. Where engagement traditions can be viewed neutrally, negatively, or as sexist, many heterosexual males and females view the "old-fashioned" approach positively as a way to concretize the commitment between two people recognizable to themselves, to their friends and family, and to the public (Schweingruber et. al., 2004, p. 148). Men expressed logical reasons for being the ones to initiate the proposal, such as an unspoken expectation to provide an engagement ring, and some women expressed that her readiness was the very reason she needed to wait for his initiation: So that she would know that he was ready too (Schweinguber et. al., 2004). This emphasizes that once physical and emotional connections have been made, it is women that are typically more ready for commitment even though men are expected to be the ones to propose it. Further, a marriage proposal itself is not only a formal request for a long-term commitment but an expression of emotional intimacy: a male-first task.

Men and women experience marriage differently despite a meta-analysis done by Jeffrey et. al. (2014) showing the difference in marital satisfaction to be very small (p. 111). The authors bring up several good points regarding why the data may be showing this is the case when it is most common to hear how marriage is a more satisfying, supportive experience for men that it is for women. One of these reasons has to do with data differences between groups of men and women in the community compared to men and women in marital therapy in this study. For the latter, the research shows females to be " $51 \%$ less likely to be satisfied with their" marriage compared to males whereas the community participants were close showing equal levels of satisfaction between the sexes (Jeffrey et. al., 2014, p. 115). Pertaining to the leveling out of marital satisfaction in relationships more recently has to do with the increased acceptance for women to exit marriage contracts that are no longer fulfilling for them as they are increasingly less dependent on marriage for practical purposes. More commonly, it is for reasons of love and emotional connection that women seek out commitment in relationships such as marriage. This could have something to do with the fact that women are $73 \%$ more likely to initiate marital counselling, indicating that once the initiatory practices that men take to build a physical, emotional, and longerterm connection, the maintenance requirements of a committed relationship seem to faulter somewhat and it is women that are more inclined to seek help to fix or save the relationship (Jeffrey et, al., 2014). Those in marital counselling show not only more dissatisfied women, but more satisfied men indicating there is likely a difference in relationship expectations in long-term relationships such as marriage. For those equally dissatisfied, men reported struggling with juggling the demands of work, meeting wives emotional and communication needs, being a parent, and meeting their own sexual needs. When these things fail alongside an attempt to save the relationship, it is women that more commonly initiate relationship dissolution and divorce, regardless of who is at fault (Jeffrey et. al., 2014). 


\section{Benevolent Sexism}

Sexism usually connotes negative attitudes towards women, both discriminatory and prejudicial (American Psychological Association, 2020). This refers to the hostility we commonly associate with sexism. Benevolent sexism, on the other hand, holds a positive connotation specific to women held by both sexes. According to the study done by Robnett et. al. in 2013, benevolent sexism is reflected in the attitudes of men and women, young and old, when it comes to the traditional engagement proposal, also to the tradition of surname changes upon marriage. The participants were young; the findings of this study are important because of this fact. Caught somewhere between young adults and the Baby boomer generation, I personally see hostile sexism more so in older, conservative generations. This study shows the positive spin of sexism in relation to marriage proposals in line with the results of the research in Schweingruber et. al.'s article where "old-fashioned" traditions are viewed positively, also in young, university aged young adults (2004, p. 148). Where surname changes used to be mandatory by the law and represented the ownership of a woman by her husband, this is no longer the case. However, the preference for maintaining this tradition as well as traditional marriage proposals continue, even among young people of a liberal mindset who demonstrate knowledge, choice, and agency over the situation. In fact, researchers have found that there is a perception of weakness in couples who lacked two marital traditions: the man proposing on one knee with a diamond ring, and her accepting his surname as her own (Robnett et. al., 2013).

Where taking a man's surname upon marriage was previously an act of power and control over women, women now report doing so willingly and is an expression of their personal desire. To them, changing their name is for reasons of maintaining tradition, practicality, and the associated symbolism of love, respect, and unity (Robnett et. al., 2013). While benevolent sexism can be viewed as a gentle, covert way of maintaining control and power over women through traditional courtship and marriage practices, I disagree that this is the case. While there has been a focus on the preference for men to initiate the engagement, researchers also found that on the flip side that not a single woman or man in their participant group "definitely" wanted a woman to be the proposer (Robnett et. al., 2013, p. 104). They also found that both sexes preferred tradition and romance as catalysts for maintaining these gender roles; strictly women reported wanting to maintain a level of comfort which I associate with the relaxed stance of feminine receptivity; and men reported a desire to maintain and utilize their decision capabilities (Robnett et. al., 2013). All of this reflects that both women and men are advocating for the maintenance of gender-roles because they value the essence of what it means to be fundamentally feminine and masculine while in relationship with the opposite sex. This does not take away from women's ability to have agency or make decisions, nor does it take away from men's ability to let go and receive. It simply indicates there is an interplay between masculine and feminine traits in heterosexual relationships that is valued and preferred even when given a choice not to play by traditional relationship standards. Further, to think that a woman is somehow losing her identity when she decides to wed and take her partner's surname is to reduce this choice to either-or thinking. The changing of a surname indicates a transition of woman's marital status which has likely impacted her personal identity: It symbolizes her receptivity of this transition and equally reflects his potency. I also argue that when a man asks for a woman's hand in marriage, the choice is not solely his but hers 
as well: She has every right to decline his invitation. To reduce a traditional marriage proposal and surname change to a game of power and control misses bigger picture in which rituals such as these serve to provide meaning between men and women in an otherwise ordinary life.

\section{Evolutionary Theory}

As discussed thus far, the research shows a tendency for males to be more direct, comfortable, confident, and frequent in initiating intimate relationships whether short-term or long-term, where women are more comfortable taking a passive or receptive role through image dependence and displaying emotional warmth (Clark et. al., 1999). This suggests that women have the need to be more cautious than do men and shares the perspective of evolutionary theories. In a world where women are quite capable at surviving and thriving on their own, especially in Western cultures, and where it is not uncommon to support a family by themselves, women are biologically programmed to be discerning of who they form intimate relationships with. Thus, while women are excelling and sometimes surpassing their male counterparts in the external world of career, inside the home and personal sphere there is a preference and degree of comfort on the part of both men and women to revert to heteronormative gender roles. While relationship initiation on the part of males is strongly related to a psychological identification with male gender-roles, i.e., masculinity, there is also a strong argument for the role of biology: Women are physically and physiologically built to receive the male phallus and his reproductive gifts. Men come first, quite literally. From the beginnings of mankind according to Genesis in the Bible to the research done by Clark et. al. (1999) and Harrison et. al., (2011), men score first place also in readiness for sex, recognizing feelings of love, and verbalizing the statement "I love you" to their female partners. This follows the evolutionary perspective in that it indicates females exercise their discretion in mate selection. It is to their reproductive advantage to wait, to let the male display his own unique set of strengths and vulnerabilities by reaching out and pursuing a woman through actions, words, and emotional sentiments. Even though women continue to gain more independence, when it comes to partner selection there are biological factors deeply engrained generationally whereby females have a preference and instinct to follow, to let their partners lead, and to make choices that are right for them reproductively whether conscious or not.

Furthering the importance biology has on gender role manifestation are jealousy responses produced particularly in males. Where young men become jealous at the thought of their female partners engaging in sex with other males, females expressed more jealousy at the thought of their male partners engaging with other females on an emotional level (Larsen et. al., 2021). The participants in this study were young, and so the question as to why adolescents demonstrate these emotional responses when neither girls nor boys are ready psychologically to marry or parent children remains (Larsen et. al., 2021). It is an instinctual response that exists well before the commitment stage of a relationship demonstrating that women, no matter their age, are protective of their attachment to males whereby losing him to another woman signals high fitness costs in the same way that men's jealousy protects their reproductive investment (Larsen et. al., 2021). While reading, I began thinking of the tradition of giving a diamond engagement ring during a marriage proposal. Given a diamond's value, this tradition could be seen a physical manifestation of a cul- 
tural ritual where the deeper biological meaning indicates a signal to other men that this woman is spoken for without having to reduce himself to aggressive measures to ward others away. The diamond ring symbolizes emotional attachment, sexual fidelity, mutual commitment, and financial security.

\section{Conclusion}

While gender roles could be viewed as being prescribed by the rules set out by society, reinforced through media and popular culture, the maintenance of these gender roles in intimate heterosexual relationships is the result of other factors. The preference for male initiation in the physical, emotional, and commitment realms of intimate relationships stems from the unique biology of males and females. Particular to engagement tradition and surname changes, a cognitive choice based is made based on the value perceived in maintaining these practices where differences between the sexes is viewed not as a threatening power differential but an expression of the value and equality of both men and women and their inter-dependent roles in relation to each other. 


\section{References}

American Psychological Association. (2020). In APA dictionary of psychology. Retrieved November 11, 2021, from https://dictionary.apa.org

Clark, C. L., Shaver, P. R., \& Abrahams, M. F. (1999). Strategic behaviors in romantic relationship initiation. Personality \& Social Psychology Bulletin, 25(6), 707. https://doi.org/10.1177/0146167299025006006

Eryilmaz, A., \& Atak, H. (2011). Investigation of starting romantic intimacy in emerging adulthood in terms of self-esteem, gender and gender roles. Educational Sciences: Theory and Practice, 11(2), 595-600.

Grøntvedt, T. V., Kennair, L. E. O., \& Mehmetoglu, M. (2015). Factors predicting the probability of initiating sexual intercourse by context and sex. Scandinavian Journal of Psychology, 56(5), 516-526. https://doi.org/10.1111/sjop.12215

Harrison, M., \& Shortall, J. (2011). Women and men in love: Who really feels it and says it first? Journal of Social Psychology, 151(6), 727-736. https://doi.org/10.1080/00224545.2010.522626

Jeffrey B. Jackson, Richard B. Miller, Megan Oka, \& Ryan G. Henry. (2014). Gender differences in marital satisfaction: A meta-analysis. Journal of Marriage and Family, 76(1), 105-129.

Larsen, P. H. H., Bendixen, M., Grøntvedt, T. V., Kessler, A. M., \& Kennair, L. E. O. (2021). Investigating the emergence of sex differences in jealousy responses in a large community sample from an evolutionary perspective. Scientific Reports, 11(1), 1-9. https://doi.org/10.1038/s41598-021-85997-7

Robnett, R. D., \& Leaper, C. (2013). 'Girls don't propose! Ew': A mixed-methods examination of marriage tradition preferences and benevolent sexism in emerging adults. Journal of Adolescent Research, 28(1), 96-121. https://doi.org/10.1177/0743558412447871

Schweingruber, D., Anahita, S., \& Berns, N. (2004). "Popping the question" When the answer is known: The engagement proposal as performance. Sociological Focus, 37(2), 143-161. https://doi.org/10.1080/00380237.2004.10571239 